\title{
Homeomorphism between Sphere and Cube
}

\author{
Amidora Idris $^{1, *}$, Tahir Ahmad ${ }^{2}$, Normah Maan ${ }^{1}$ \\ ${ }^{1}$ Fuzzy Research Group, Department of Mathematics, Faculty of Science, Universiti Teknologi Malaysia, 81310 Skudai, Johor, \\ Malaysia \\ ${ }^{2}$ Ibnu Sina Institute for Fundamental Science Studies, Universiti Teknologi Malaysia, 81310 Skudai, Johor, Malaysia \\ *Author to whom correspondence should be addressed. E-mail: amidora@mel.fs.utm.my
}

Received: 31 October 2008

http://dx.doi.org/10.11113/mjfas.v4n2.42

ABSTRACT

In this paper, we present the construction of homeomorphism from unit sphere; $S^{2}$ to unit cube; $C^{3}$. On route, we produced an explicit mapping between the two topological spaces where proving by construction is mainly adopted in this work.

| Homeomorphism | Homeomorphic | Continuously deformed |

\section{Introduction}

Sphere and cube are two different geometrical objects. Even though a sphere and a cube have a different shape, topologically there is no difference between them [1]. In that case, we can say that the surface of a sphere is a topologically equivalent to the surface of a cube. Two structures are topologically equivalent if and only if one shape can be continuously deformed to the other shape such as bending, stretching or squeezing without being severed, tearing or gluing [2]. In other words, there exists homeomorphism that is an open continuous bijection mapping between sphere and cube.

We consider two surfaces in space: a sphere and a cube. We can see that the sphere can be continuously deformed into the cube without tearing or collapsing them [3]. Many literature [3], [4], [5], [6], [7] mentioned that it is not hard to convince that a sphere can be deformed into a cube. However not all of them come up with an explicit mapping for a homeomorphism between them. Even though J.M. Lee in [3] posted the mapping from a sphere to a cube, he left out most of the essentials proofs to be homeomorphics.

Therefore, in this paper we construct the homeomorphism between unit sphere, $S^{2}$ and unit cube, $C^{3}$. This paper is organized into five sections. In the next section, we define $C^{3}$ and prove that it is a topological space. The proof for $S^{2}$ was left out because Tahir in [8] had proved that it is a topological space. In section 3, we construct and define the mapping explicitly from $S^{2}$ to $C^{3}$ and built up several related lemmas. Section 4 , we prove the homeomorphism between them by showing openness, bijective and continuity of the mapping. Finally, we draw some conclusions in the last section of this paper. 


\section{2. $C^{3}$ as a topological space}

Before we prove that $C^{3}$ is a topological space, we defined the cube $C^{3}$ as follows. We denote $C^{3}$ as a surface of the unit cube $[-1,1]^{3} \backslash(-1,1)^{3}$ bounded by six square faces centered at the origin in three-dimension (Figure 1).

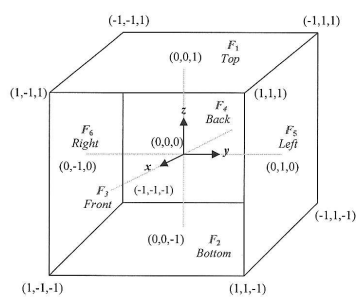

Figure 1: $C^{3}$

Next, we show that $C^{3}$ is a topological space as follows. We define the open set $V$ on $C^{3}$ as $V=\theta \cap C^{3}$ where $\theta$ is an open set in $R^{3}$. Suppose $\tau_{C^{3}}$ is a collection of all open sets of $C^{3}$.

First, we need to show that $\varnothing \in \tau_{C^{3}}$ and $C^{3} \in \tau_{C^{3}}$. We know that $\varnothing$ is a subset of any set [9] and it is open [10]. Therefore $\varnothing$ is an open set for $C^{3}$. Thus, $\varnothing \in \tau_{C^{3}}$. We also know that $R^{3}$ is open and $C^{3}$ is a subset of $R^{3}$. Thus $R^{3} \cap C^{3}=C^{3}$ is an open set for $C^{3}$. Therefore, $C^{3} \in \tau_{C^{3}}$.

Next, we show that $\bigcup_{i \in I} V_{i} \in \tau_{C^{3}}$ where $V_{i}=\theta_{i} \cap C^{3}, \theta_{i}$ is an open set in $R^{3}$. Notice that

$$
\begin{aligned}
\bigcup_{i \in I} V_{i} & =V_{1} \cup V_{2} \cup V_{3} \cup V_{4} \cup \ldots \cup V_{i-1} \cup V_{i} \cup \ldots \\
& =\left(\theta_{1} \cap C^{3}\right) \cup\left(\theta_{2} \cap C^{3}\right) \cup\left(\theta_{3} \cap C^{3}\right) \cup\left(\theta_{4} \cap C^{3}\right) \cup \ldots \\
& =\left[\left(\theta_{1} \cup \theta_{i-1} \cap C^{3} \cup \theta_{3} \cup \theta_{i} \cap C_{4}^{3} \cup \ldots \ldots\right.\right. \\
& =\left(\bigcup_{i \in I} \theta_{i}\right) \cap C^{3} .
\end{aligned}
$$

Since $\bigcup_{i \in I} \theta_{i}$ is open in $R^{3}$, thus $\bigcup_{i \in I} V_{i}=\left(\bigcup_{i \in I} \theta_{i}\right) \cap C^{3}$ is open in $C^{3}$. Therefore, $\bigcup_{i \in I} V_{i} \in \tau_{C^{3}}$.

Lastly, we show that $\bigcap_{i=I}^{n} V_{i} \in \tau_{C^{3}}$. Notice that

$$
\begin{aligned}
\bigcap_{i=I}^{n} V_{i} & =V_{1} \cap V_{2} \cap V_{3} \cap V_{4} \cap \ldots \cap V_{n-1} \cap V_{n} \\
& =\left(\theta_{1} \cap C^{3}\right) \cap\left(\theta_{2} \cap C^{3}\right) \cap\left(\theta_{3} \cap C^{3}\right) \cap\left(\theta_{4} \cap C^{3}\right) \cap \ldots \\
& =\left[\left(\theta_{1} \cap \theta_{n-1} \cap C^{3}\right) \cap\left(\theta_{n} \cap C^{3}\right)\right. \\
& \left.\left.=\left(\bigcap_{i=1}^{n} \theta_{i}\right) \cap \theta_{4} \cap \ldots \cap \theta_{n-1} \cap \theta_{n}\right)\right] \cap C^{3}
\end{aligned}
$$


Since $\left(\bigcap_{i=1}^{n} \theta_{i}\right)$ is open in $R^{3}$, thus $\bigcap_{i=I}^{n} V_{i}=\left(\bigcap_{i=1}^{n} \theta_{i}\right) \cap C^{3}$ is open in $C^{3}$. Therefore, $\bigcap_{i=I}^{n} V_{i} \in \tau_{C^{3}}$. Hence, $\tau_{C^{3}}$ is indeed a topology on $C^{3}$ and thus $\left(C^{3}, \tau_{C^{3}}\right)$ is a topological space.

In the next section, the construction of mapping between unit sphere, $S^{2}$ to unit cube, $C^{3}$ will be given.

\section{Mapping from $S^{2}$ to $C^{3}$}

In this section, we define the mapping from $S^{2}$ to $C^{3}$ as follows:

$\varnothing: S^{2} \rightarrow C^{3}$ (Figure 2) such that

$$
\varnothing(x, y, z)=\frac{(x, y, z)}{\max \{|x|,|y|,|z|\}}
$$

where $S^{2}=\left\{(x, y, z) \in R^{3}: x^{2}+y^{2}+z^{2}=1\right\}$ and $C^{3}=[-1,1]^{3} \backslash(-1,1)^{3}$.
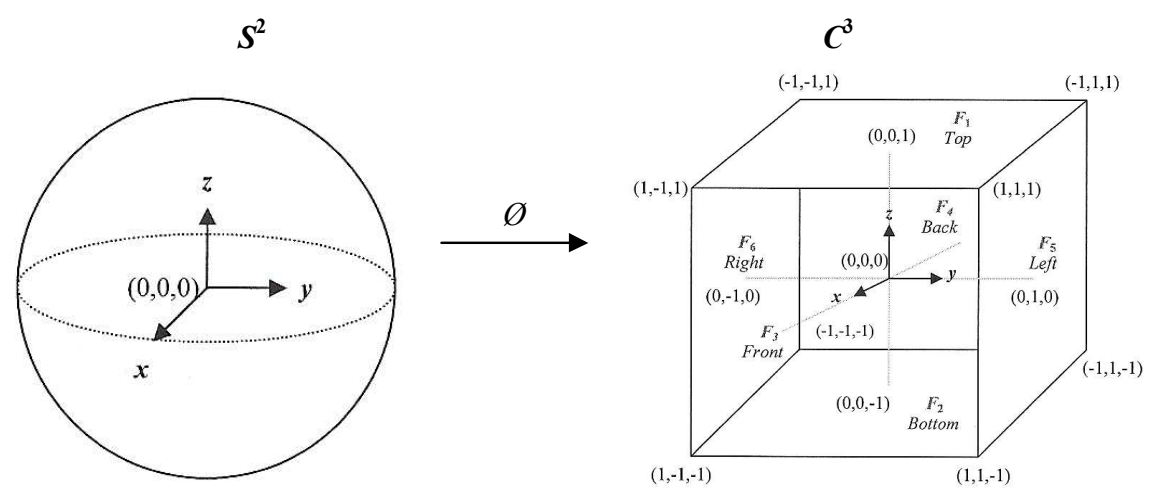

Figure 2: The mapping from $S^{2}$ to $C^{3}$

Next, we partition the unit sphere as $S^{2}=\bigcup_{i=1}^{6} D_{i}$ into six regions as below :

$$
\begin{aligned}
& D_{1}:\left\{(x, y, z) \in S^{2}: \frac{1}{\sqrt{3}} \leq z \leq 1,-\frac{1}{\sqrt{3}} \leq x, y \leq \frac{1}{\sqrt{3}}, z \geq x, y\right\}, \\
& D_{2}:\left\{(x, y, z) \in S^{2}:-1 \leq z \leq-\frac{1}{\sqrt{3}},-\frac{1}{\sqrt{3}} \leq x, y \leq \frac{1}{\sqrt{3}},|z| \geq x, y\right\}, \\
& D_{3}:\left\{(x, y, z) \in S^{2}: \frac{1}{\sqrt{3}} \leq x \leq 1 \quad,-\frac{1}{\sqrt{3}}<y, z<\frac{1}{\sqrt{3}}, x>y, z\right\}, \\
& D_{4}:\left\{(x, y, z) \in S^{2}:-1 \leq x \leq-\frac{1}{\sqrt{3}} \quad,-\frac{1}{\sqrt{3}}<y, z<\frac{1}{\sqrt{3}},|x|>y, z\right\},
\end{aligned}
$$




$$
\begin{aligned}
& D_{5}:\left\{(x, y, z) \in S^{2}: \frac{1}{\sqrt{3}} \leq y \leq 1 \quad,-\frac{1}{\sqrt{3}} \leq x \leq \frac{1}{\sqrt{3}},-\frac{1}{\sqrt{3}}<z<\frac{1}{\sqrt{3}}, y \geq x, y>z\right\} \\
& D_{6}:\left\{(x, y, z) \in S^{2}:-1 \leq y \leq-\frac{1}{\sqrt{3}},-\frac{1}{\sqrt{3}} \leq x \leq \frac{1}{\sqrt{3}},-\frac{1}{\sqrt{3}}<z<\frac{1}{\sqrt{3}},|y| \geq x,|y|>z\right\} .
\end{aligned}
$$

On the other hand, we partition the unit cube as $C^{3}=\bigcup_{i=1}^{6} F_{i}$ (Figure 1) where

$$
\begin{aligned}
& F_{1}:\left\{(x, y, z) \in C^{3}: z=1,-1 \leq x, y \leq 1\right\}, \\
& F_{2}:\left\{(x, y, z) \in C^{3}: z=-1,-1 \leq x, y \leq 1\right\}, \\
& F_{3}:\left\{(x, y, z) \in C^{3}: x=1,-1<y, z<1\right\}, \\
& F_{4}:\left\{(x, y, z) \in C^{3}: x=-1,-1<y, z<1\right\}, \\
& F_{5}:\left\{(x, y, z) \in C^{3}: y=1,-1 \leq x \leq 1,-1<z<1\right\}, \\
& F_{6}:\left\{(x, y, z) \in C^{3}: y=-1,-1 \leq x \leq 1,-1<z<1\right\} .
\end{aligned}
$$

So, the mapping can be redefined as :

$$
\varnothing: \bigcup_{i=1}^{6} D_{i}=S^{2} \rightarrow \bigcup_{i=1}^{6} F_{i}=C^{3}
$$

Then, we build the following lemmas in order to prove that $S^{2}$ is homeomorphic to $C^{3}$.

Lemma 3.1 If $x, y \in[-a, a]$ and $z \in[a, 1]$ for $0 \leq a \leq \frac{1}{\sqrt{3}}$, then $\max \{|x|,|y|,|z|\}=z$.

Proof :

Consider $S=\left\{(x, y, z): x, y \in[-a, a]\right.$ and $z \in[a, 1]$ for $\left.0 \leq a \leq \frac{1}{\sqrt{3}}\right\}$.

Therefore $\max \{|x|\}=\max \{|y|\}=a$ and $\min \{|z|\}=a$.

Observed that,

$$
\max \{|x|,|y|,|z|\} \geq \max \{|x|\}=a \text { and } \max \{|x|,|y|,|z|\} \geq \max \{|y|\}=a .
$$

But then,

$$
\max \{|x|,|y|,|z|\} \geq \max \{|z|\} \geq \min \{|z|\}=a=\max \{|x|\}=\max \{|y|\} .
$$

Then, if $\left(x_{o}, y_{o}, z_{o}\right) \in S, \max \left\{\left|x_{o}\right|,\left|y_{o}\right|,\left|z_{o}\right|\right\}=z_{o}$ since $z_{o}>0$, so $\left|z_{o}\right| \geq \min \{|z|\}, \forall z \in[a, 1]$.

\section{Lemma 3.2 If}

$D_{1}:\left\{(x, y, z) \in S^{2}: \frac{1}{\sqrt{3}} \leq z \leq 1,-\frac{1}{\sqrt{3}} \leq x, y \leq \frac{1}{\sqrt{3}}, z \geq x, y \quad\right\}$,

$F_{1}:\left\{(x, y, z) \in C^{3}: z=1,-1 \leq x, y \leq 1\right\}$ and there exists a mapping

$\varnothing: D_{1} \rightarrow F_{1} \ni \varnothing(x, y, z)=\frac{(x, y, z)}{\max \{|x|,|y|,|z|\}}$, then $\varnothing$ is onto. 
Proof :

We will prove that $\varnothing\left(D_{1}\right)=F_{1}$ by showing that $\varnothing\left(D_{1}\right) \subseteq F_{1}$ and $F_{1} \subseteq \varnothing\left(D_{1}\right)$, where $\varnothing(x, y, z)=\left(x^{\prime}, y^{\prime}, z^{\prime}\right) \in F_{1}$ for $(x, y, z) \in D_{l}$.

(i) $\quad \varnothing\left(D_{1}\right) \subseteq F_{1}$

We choose $a \in \emptyset\left(D_{1}\right)$, therefore $a=\frac{(x, y, z)}{\max \{|x|,|y|,|z|\}}$ for some $(x, y, z) \in D_{l}$.

By Lemma 3.1, we have $\max \{|x|,|y|,|z|\}=z$.

Therefore $a=\frac{(x, y, z)}{z}=\left(\frac{x}{z}, \frac{y}{z}, 1\right), z \neq 0$. Then, we need to show $-1 \leq \frac{x}{z}, \frac{y}{z} \leq 1$

But, $\quad \frac{-1 / \sqrt{3}}{1} \leq \frac{x}{z} \leq \frac{1 / \sqrt{3}}{1 / \sqrt{3}} \quad \Rightarrow \quad-\frac{1}{\sqrt{3}} \leq \frac{x}{z} \leq 1$.

Similarly, $\quad \frac{-1 / \sqrt{3}}{1} \leq \frac{y}{z} \leq \frac{1 / \sqrt{3}}{1 / \sqrt{3}} \Rightarrow-\frac{1}{\sqrt{3}} \leq \frac{y}{z} \leq 1$.

Thus $a \in F_{1}$.

(ii) $\quad F_{1} \subseteq \varnothing\left(D_{1}\right)$

We choose $b \in F_{1}$. Therefore $b=\left(x^{\prime}, y^{\prime}, 1\right) \ni x^{\prime} \in[-1,1]$ and $y^{\prime} \in[-1,1]$.

We need to show that $b \in \varnothing\left(D_{1}\right)$.

$$
\begin{aligned}
& \text { But then }\left(x^{\prime}, y^{\prime}, 1\right)=\frac{\left(x^{\prime} z, y^{\prime} z, z\right)}{z} \quad \text { for } \frac{1}{\sqrt{3}} \leq z \leq 1, z \neq 0 \text { and }-\frac{1}{\sqrt{3}} \leq x^{\prime} z, y^{\prime} z \leq \frac{1}{\sqrt{3}} \\
& =\frac{\left(x^{\prime} z, y^{\prime} z, z\right)}{\max \left\{\left|x^{\prime} z\right|,\left|y^{\prime} z\right|,|z|\right\}} \\
& =\frac{(x, y, z)}{\max \{|x|,|y|,|z|\}} \quad \text { where } \quad x=x^{\prime} z, y=y^{\prime} z \\
& =\varnothing(x, y, z) \quad \text { for } \frac{1}{\sqrt{3}} \leq z \leq 1 \text { and }-\frac{1}{\sqrt{3}} \leq x, y \leq \frac{1}{\sqrt{3}} \\
& =\varnothing\left(D_{1}\right) .
\end{aligned}
$$

Thus, $b \in \varnothing\left(D_{1}\right)$.

Since $\varnothing\left(D_{l}\right) \subseteq F_{l}$ and $F_{l} \subseteq \varnothing\left(D_{l}\right)$, clearly $\varnothing\left(D_{l}\right)=F_{l}$.

By, using similar arguments as shown in Lemma 3.1 and Lemma 3.2, we can have the following Lemmas for the other five partitions respectively. 
Lemma 3.3 If $x, y \in[-a, a]$ and $z \in[-1,-a]$ for $0 \leq a \leq \frac{1}{\sqrt{3}}$, then $\max \{|x|,|y|,|z|\}=|z|$.

Lemma 3.4 If

$D_{2}:\left\{(x, y, z) \in S^{2}:-1 \leq z \leq-\frac{1}{\sqrt{3}},-\frac{1}{\sqrt{3}} \leq x, y \leq \frac{1}{\sqrt{3}},|z| \geq x, y \quad\right\}$,

$F_{2}:\left\{(x, y, z) \in C^{3}: z=-1,-1 \leq x, y \leq 1\right\}$ and there exists a mapping

$\varnothing: D_{2} \rightarrow F_{2} \quad \ni \quad \varnothing(x, y, z)=\frac{(x, y, z)}{\max \{|x|,|y|,|z|\}}$, then $\varnothing$ is onto.

Lemma 3.5 If $y, z \in(-a, a)$ and $x \in[a, 1]$ for $0 \leq a \leq \frac{1}{\sqrt{3}}$, then $\max \{|x|,|y|,|z|\}=x$.

Lemma 3.6 If

$D_{3}:\left\{(x, y, z) \in S^{2}: \frac{1}{\sqrt{3}} \leq x \leq 1,-\frac{1}{\sqrt{3}}<y, z<\frac{1}{\sqrt{3}}, x>y, z\right\}$,

$F_{3}:\left\{(x, y, z) \in C^{3}: x=1,-1<y, z<1\right\}$ and there exists a mapping

$\varnothing: D_{3} \rightarrow F_{3} \quad \ni \varnothing(x, y, z)=\frac{(x, y, z)}{\max \{|x|,|y|,|z|\}}$, then $\varnothing$ is onto.

Lemma 3.7 If $y, z \in(-a, a)$ and $x \in[-1,-a]$ for $0 \leq a \leq \frac{1}{\sqrt{3}}$, then $\max \{|x|,|y|,|z|\}=|x|$.

Lemma 3.8 If

$D_{4}:\left\{(x, y, z) \in S^{2}:-1 \leq x \leq-\frac{1}{\sqrt{3}},-\frac{1}{\sqrt{3}}<y, z<\frac{1}{\sqrt{3}},|x|>y, z\right\}$,

$F_{4}:\left\{(x, y, z) \in C^{3}: x=-1,-1<y, z<1\right\}$ and there exists a mapping

$\varnothing: D_{4} \rightarrow F_{4} \quad \ni \quad \varnothing(x, y, z)=\frac{(x, y, z)}{\max \{|x|,|y|,|z|\}}$, then $\varnothing$ is onto.

Lemma 3.9 If $x \in[-a, a], z \in(-a, a)$ and $y \in[a, 1]$ for $0 \leq a \leq \frac{1}{\sqrt{3}}$, then $\max \{|x|,|y|,|z|\}=y$.

\section{Lemma 3.10 Let}

$D_{5}:\left\{(x, y, z) \in S^{2}: \frac{1}{\sqrt{3}} \leq y \leq 1,-\frac{1}{\sqrt{3}} \leq x \leq \frac{1}{\sqrt{3}},-\frac{1}{\sqrt{3}}<z<\frac{1}{\sqrt{3}}, y \geq x, y>z\right\}$,

$F_{5}:\left\{(x, y, z) \in C^{3}: y=1,-1 \leq x \leq 1,-1<z<1\right\}$ and there exists a mapping

$\varnothing: D_{5} \rightarrow F_{5} \quad \ni \varnothing(x, y, z)=\frac{(x, y, z)}{\max \{|x|,|y|,|z|\}}$, then $\varnothing$ is onto.

Lemma 3.11 If $x \in[-a, a], z \in(-a, a)$ and $y \in[-1,-a]$ for $0 \leq a \leq \frac{1}{\sqrt{3}}$, then $\max \{|x|,|y|,|z|\}=|y|$. 
Lemma 3.12 If

$D_{6}:\left\{(x, y, z) \in S^{2}:-1 \leq y \leq-\frac{1}{\sqrt{3}},-\frac{1}{\sqrt{3}} \leq x \leq \frac{1}{\sqrt{3}},-\frac{1}{\sqrt{3}}<z<\frac{1}{\sqrt{3}}, y \geq x,|y|>z\right\}$,

$F_{6}:\left\{(x, y, z) \in C^{3}: y=-1,-1 \leq x \leq 1,-1<z<1\right\}$ and there exists a mapping

$\varnothing: D_{6} \rightarrow F_{6} \ni \varnothing(x, y, z)=\frac{(x, y, z)}{\max \{|x|,|y|,|z|\}}$, then $\varnothing$ is onto.

In this section, we have shown that $\varnothing$ is a function from $\bigcup_{i=1}^{6} D_{i}=S^{2}$ to $\bigcup_{i=1}^{6} F_{i}=C^{3}$ and we are now ready to show the homeomorphism between them in the following section.

\section{Homeomorphism between $S^{2}$ and $C^{3}$}

In this section, we will prove the equivalent structure of the sphere and cube (i.e. homeomorphism). In other words, $S^{2} \cong C^{3}$. Before that, we present some general definitions and theorems which will be used along with the construction of this homeomorphism.

Theorem 4.1 [11] If $F=\left(f_{1}, f_{2}, \ldots, f_{m}\right)$ is a mapping from $R^{n}$ to $R^{m}$, then

$$
F_{*}\left(U_{j}(p)\right)=\sum_{i=1}^{m} \frac{\partial f_{i}}{\partial x_{j}}(p) U_{i}(F(p)), \quad \text { for } 1 \leq j \leq n .
$$

Jacobian matrix of $F$ at $p$ is

$$
\left(\left(\frac{\partial f_{i}}{\partial x_{j}}(p)\right)\right), \quad \text { for } 1 \leq i \leq m, 1 \leq j \leq n
$$

Theorem 4.2 [9] A map $f: X \rightarrow Y$ from topological space $X$ to a topological space $Y$ is continuous if and only if the inverse image $f^{l}(U)$ of each open subset $V$ of $Y$ is open in $X$.

Definition 4.3 [9] A map $f: X \rightarrow Y$ between topological space is open if the image $f(U)$ of each open subset $U$ of $X$ is open in $Y$ and closed if the image $f(E)$ of each closed subset $E$ of $X$ is closed in $Y$.

Definition 4.4 [12] A function $f: X \rightarrow Y$ between topological space is called a homeomorphism if $f: X \rightarrow Y$ is one-to-one and onto and both $f$ and $f^{1}$ are continuous. The notation $X \cong Y$ means that $X$ is homeomorphic to $Y$.

Theorem 4.5 $\quad S^{2}$ is homeomorphic to $C^{3}$

Proof :

Firstly, we define $\varnothing: S^{2} \rightarrow C^{3} \ni \varnothing(x, y, z)=\frac{(x, y, z)}{\max \{|x|,|y|,|z|\}}$ with

$S^{2}=\left\{(x, y, z) \in R^{3}: x^{2}+y^{2}+z^{2}=1\right\}$ and $C^{3}=[-1,1]^{3} \backslash(-1,1)^{3}$. 
Assume $\left(x_{1}, y_{1}, z_{1}\right)=\left(x_{2}, y_{2}, z_{2}\right)$. Therefore $\frac{\left(x_{1}, y_{1}, z_{1}\right)}{\max \left\{\left|x_{1}\right|,\left|y_{1}\right|,\left|z_{1}\right|\right\}}=\frac{\left(x_{2}, y_{2}, z_{2}\right)}{\max \left\{\left|x_{2}\right|,\left|y_{2}\right|,\left|z_{2}\right|\right\}}$ which implies $\varnothing\left(x_{1}, y_{1}, z_{1}\right)=\varnothing\left(x_{2}, y_{2}, z_{2}\right)$. Thus, $\varnothing$ is a function from a topological space $S^{2}$ to a topological space $C^{3}$. In the following proving, we will consider the function as $\varnothing: \bigcup_{i=1}^{6} D_{i}=S^{2} \rightarrow \bigcup_{i=1}^{6} F_{i}=C^{3}$.

Next, we will show $\varnothing$ is bijective. Pick $a \in C^{3}$. Therefore $\exists(x, y, z) \in S^{2} \ni a=\frac{(x, y, z)}{\max \{|x|,|y|,|z|\}}$ $=\varnothing(x, y, z)$. Thus $\varnothing$ is onto.

Now, we will use the same proving technique that had been done by Tahir [8], [13 ] to show that $\varnothing$ is one to one. Let $\varnothing=\varnothing^{*}$ and

$$
\begin{aligned}
\varnothing(x, y, z) & =\frac{(x, y, z)}{\max \{|x|,|y|,|z|\}}=\frac{(x, y, z)}{p}=\left(\frac{x}{p}, \frac{y}{p}, \frac{z}{p}\right) \\
& =\left(\frac{x^{*}}{p^{*}}, \frac{y^{*}}{p^{*}}, \frac{z^{*}}{p^{*}}\right)=\frac{\left(x^{*}, y^{*}, z^{*}\right)}{p^{*}}=\frac{\left(x^{*}, y^{*}, z^{*}\right)}{\max \left\{\left|x^{*}\right|,\left|y^{*}\right|,\left|z^{*}\right|\right\}}=\varnothing\left(x^{*}, y^{*}, z^{*}\right)
\end{aligned}
$$

where $p, p^{*} \neq 0$ for $(x, y, z),\left(x^{*}, y^{*}, z^{*}\right) \in S^{2}$ with corresponding $p$ and $p^{*}$. Since $\varnothing(x, y, z)=\varnothing\left(x^{*}, y^{*}, z^{*}\right)$, so Jacobian matrix $\emptyset$ on $(x, y, z)=$ Jacobian matrix $\emptyset$ on $\left(x^{*}, y^{*}, z^{*}\right)$. Using Theorem 4.1, we get

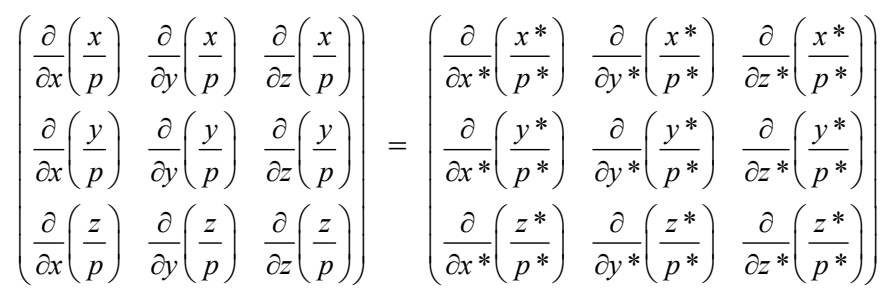

$$
\begin{aligned}
& \Rightarrow \quad\left(\begin{array}{ccc}
\frac{1}{p} & 0 & 0 \\
0 & \frac{1}{p} & 0 \\
0 & 0 & \frac{1}{p}
\end{array}\right)=\left(\begin{array}{ccc}
\frac{1}{p^{*}} & 0 & 0 \\
0 & \frac{1}{p^{*}} & 0 \\
0 & 0 & \frac{1}{p^{*}}
\end{array}\right)
\end{aligned}
$$

From (3), we have

$$
\frac{1}{p}=\frac{1}{p^{*}} \Rightarrow p=p^{*}
$$

From the components of Jacobian matrix above, we can conclude that

$$
\frac{1}{p}=\frac{1}{p^{*}} \Rightarrow p=p^{*} .
$$

This implies $\frac{x}{p}=\frac{x^{*}}{p} \Rightarrow x=x^{*}, \frac{y}{p}=\frac{y^{*}}{p} \Rightarrow y=y^{*}$ and $\frac{z}{p}=\frac{z^{*}}{p} \Rightarrow z=z^{*}$. 
Thus, $\varnothing$ is one-to-one. Since $\varnothing$ is onto and one-to-one, therefore $\varnothing$ is a bijection.

Next, we will show that $\varnothing$ is continuous by using Theorem 4.2. According to this theorem, we need to show that $\varnothing^{-1}(\beta)$ is open in $S^{2}$ for every open set $\beta$ in $C^{3}$. Pick $(a, b, c) \in \varnothing^{-1}(\beta)$ where $\beta$ is an open set in $C^{3}$. Consider

$$
\varnothing^{-1}(\beta)=\left\{\varnothing^{-1}\left(x^{\prime}, y^{\prime}, z^{\prime}\right):\left(x^{\prime}, y^{\prime}, z^{\prime}\right) \in \beta\right\} .
$$

Since $\varnothing^{-1}(\beta)$ is a bijection, there exists unique $\left(x^{\prime}, y^{\prime}, z^{\prime}\right) \in \beta$ such that $\varnothing^{-1}\left(x^{\prime}, y^{\prime}, z^{\prime}\right)=(a, b, c)$. Since $\beta$ is open, then $N\left(x^{\prime}, y^{\prime}, z^{\prime}\right) \subset \beta$ exists.

Consider

$$
\varnothing^{-1}\left(N\left(x^{\prime}, y^{\prime}, z^{\prime}\right)\right)=\left\{\varnothing^{-1}\left(x^{\prime *}, y^{\prime *}, z^{\prime *}\right):\left(x^{\prime *}, y^{\prime *}, z^{\prime *}\right) \in N\left(x^{\prime}, y^{\prime}, z^{\prime}\right)\right\} .
$$

Clearly $(a, b, c)=\varnothing^{-1}\left(x^{\prime}, y^{\prime}, z^{\prime}\right) \in \emptyset^{-1}\left(N\left(x^{\prime}, y^{\prime}, z^{\prime}\right)\right)$. Then, pick any $p \in \varnothing^{-1}\left(N\left(x^{\prime}, y^{\prime}, z^{\prime}\right)\right.$. Since $\varnothing^{-1}$ is a bijection, there exists unique $\left(x^{\prime}, y^{\prime}, z^{\prime *}\right) \in N\left(x^{\prime}, y^{\prime}, z^{\prime}\right)$ such that $\varnothing^{-1}\left(x^{\prime *}, y^{\prime *}, z^{\prime *}\right)=p$. Since $N\left(x^{\prime}, y^{\prime}, z^{\prime}\right) \subset \beta$, so $\left(x^{\prime *}, y^{\prime *}, z^{\prime *}\right) \in \beta$. Thus, $p=\varnothing^{-1}\left(x^{\prime *}, y^{\prime *}, z^{\prime *}\right) \in \varnothing^{-1}(\beta)$ and this implies $\varnothing^{-1}\left(N\left(x^{\prime}, y^{\prime}, z^{\prime}\right) \subset \varnothing^{-1}(\beta)\right.$.

Notice that

$$
(a, b, c) \in \varnothing^{-1}\left(N\left(x^{\prime}, y^{\prime}, z^{\prime}\right) \subset \varnothing^{-1}(\beta) .\right.
$$

Since $(a, b, c) \in \varnothing^{-1}(\beta)$ is arbitrary, therefore we can conclude that $(a, b, c) \in \varnothing^{-1}\left(N\left(x^{\prime}, y^{\prime}, z^{\prime}\right) \subset \varnothing^{-1}(\beta)\right.$ for every $(a, b, c) \in \varnothing^{-1}(\beta)$ for some corresponding $\left(x^{\prime}, y^{\prime}, z^{\prime}\right) \in \beta$. Hence, $\emptyset^{-1}(\beta)$ is open. Since $\beta$ is arbitrary, therefore we can conclude that $\varnothing^{-1}(\beta)$ is open in $S^{2}$ for every open set $\beta$ in $C^{3}$. Thus, by Theorem $4.2, \varnothing$ is continuous (Figure 3 ).
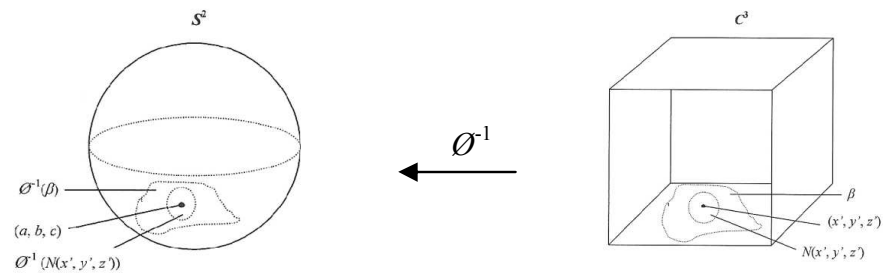

Figure 3 : $\varnothing$ is continuous

To show $\varnothing^{-1}$ is continuous is equivalent as showing $\varnothing$ is an open map. In order to prove that $\varnothing$ is an open map, by using Definition 4.3 we need to show that $\varnothing(A)$ is open in $C^{3}$ for every open set $A$ in $S^{2}$. Pick $(a, b, c) \in \emptyset(A)$ such that $A$ is an open set in $S^{2}$. Consider

$$
\varnothing(A)=\{\varnothing(x, y, z):(x, y, z) \in A\} .
$$

Since $\varnothing$ is a bijection, there exists unique $(x, y, z) \in A$ such that $\varnothing(x, y, z)=(a, b, c)$. Since $A$ is open, then $N(x, y$, $z) \subset A$ exists.

Consider

$$
\varnothing(N(x, y, z))=\left\{\varnothing\left(x^{\prime}, y^{\prime}, z^{\prime}\right):\left(x^{\prime}, y^{\prime}, z^{\prime}\right) \in N(x, y, z)\right\} .
$$

Clearly $(a, b, c)=\varnothing(x, y, z) \in \varnothing(N(x, y, z))$. Then, pick any $p \in \emptyset(N(x, y, z))$. Since $\varnothing$ is a bijection, there exists unique $\left(x^{\prime}, y^{\prime}, z^{\prime}\right) \in N(x, y, z)$ such that $\emptyset\left(x^{\prime}, y^{\prime}, z^{\prime}\right)=p$. Since $N(x, y, z) \subset A$, so $\left(x^{\prime}, y^{\prime}, z^{\prime}\right) \in A$. Thus, $p=\varnothing\left(x^{\prime}, y^{\prime}, z^{\prime}\right) \in \varnothing(A)$ and this implies $\varnothing(N(x, y, z)) \subset \varnothing(A)$.

Notice that

$$
(a, b, c) \in \emptyset(N(x, y, z)) \subset \varnothing(A) .
$$

Since $(a, b, c) \in \varnothing(A)$ is arbitrary, therefore we can conclude that $(a, b, c) \in \emptyset(N(x, y, z)) \subset \varnothing(A)$ for every $(a, b$, $c) \in \emptyset(A)$ for some corresponding $(x, y, z) \in A$. Hence, $\varnothing(A)$ is open. Since $A$ is arbitrary, therefore we conclude that $\varnothing(A)$ is open in $C^{3}$ for every open set $A$ in $S^{2}$. By using Definition $4.3, \varnothing$ is open. Therefore, $\varnothing^{-1}$ is continuous. 
Finally, since $\varnothing: S^{2} \rightarrow C^{3} \ni \varnothing(x, y, z)=\frac{(x, y, z)}{\max \{|x|,|y|,|z|\}}$ with $S^{2}=\left\{(x, y, z) \in R^{3}: x^{2}+y^{2}+z^{2}=1\right\}$ and $C^{3}=[-1,1]^{3} \backslash(-1,1)^{3}$ is onto, one-to-one, continuous as well as $\varnothing^{-1}$ is continuous, $\varnothing$ is a homeomorphism by using definition 4.4. Since homeomorphism of $S^{2}$ and $C^{3}$ exists, $S^{2}$ is homeomorphic to $C^{3}$ and we write $S^{2} \cong C^{3}$ (Figure 4).

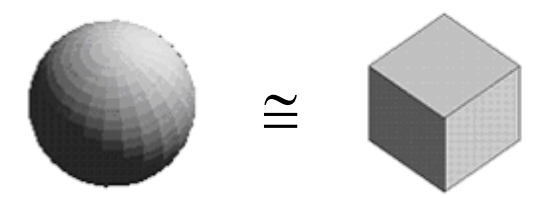

Figure 4: Homeomorphism of $S^{2}$ and $C^{3}$

\section{Conclusion}

In this paper, we have shown the construction of the homeomorphism between the unit sphere and the unit cube where they have an equivalent structure. It also preserves properties that spaces have. Results obtained from this research are very important in image processing and particularly Electroencephalography (EEG) signal for human brain.

\section{Acknowledgement}

The research is supported by Scientific Advancement Grant Allocation (SAGA) Vot No :73703. Amidora is sponsored by Universiti Teknologi Malaysia for her doctoral work. We would like also to thank the members of Fuzzy Research Group (FRG), Departments of Mathematics and Ibnu Sina Institute for Fundamental Science Studies for their assistance and cooperation.

\section{References}

[1] K.Lewin, Principles of Topological Psychology, McGraw-Hill Book Company Inc, New York, 1936.

[2] A.W. Schurle, Topics in Topology, Elsevier North Holland Inc, New York, 1979.

[3] J.M. Lee, Introduction to Topological Manifolds, Springer-Verlag, New York, 2000.

[4] N.P. Strickland, Lecture notes : Algebraic Topology, University of Sheffield, UK, 2007.

[5] E.Kasner and J.R. Newman, Mathematics and Imagination, Courier Dover Publications, New York, 2001.

[6] C.Kosniowski, The Mathematical Gazette, 62(422) (1978) 233-245.

[7] W.P. Thurston, Three-Dimensional Geometry and Topology, Princeton University Press, USA, 1997.

[8] Tahir Ahmad, The Riemann Surface: $S^{2}$, Master Thesis, California State University, 1989.

[9] M.Eisenberg, Topology, Holt, Rinehart and Winston Inc, New York, 1974.

[10] I. Abdullah, Analisis Nyata, Dewan Bahasa dan Pustaka, Kuala Lumpur, 1995.

[11] B.Oneill, Elementary Differential Geometry, Academic Press, London, 1966.

[12] G.E. Bredon, Topology and Geometry, Springer-Verlag, New York, 1993.

[13] Tahir Ahmad, Matematika, 9(1) (1993) 9-17. 